\title{
Caracterização das internações de dependentes químicos em Unidade de Internação Psiquiátrica do Hospital Geral
}

\author{
Characterization of admission of chemical-dependents \\ in a psychiatric admission unit of the General Hospital
}

Fernando Sérgio Pereira de Sousa ${ }^{1}$

Eliany $N$ azaréO liveira ${ }^{2}$

${ }^{1}$ Centro deAtenção

Psicossocial de Sobral,

Ceará. Rua Dr. Guarany

1138, Centro. 62100-000

Sobral CE.

fernando_sergio_1@

hotmail.com

${ }^{2}$ Curso de Enfermagem,

Universidade Estadual Vale

do Acaraú.

Abstract The Psychiatric Admission Unit of the General Hospital (UIPHG) is integrated to the psychiatric reform movement. The aim of this study was to evaluate chemical-dependent's admission causes at the UIPH G Dr. Estevam in the city of Sobral, Ceará State. It was a documental study with a quantitativeapproach involving 203 patients who were admitted at the UIPGH Dr. Estevam from October 2005 to A pril 2006. Data were collected from a document filled during admission processin the hospital. $95.1 \%$ of the sample was represented by men, age range of 30-40 years $(62.5 \%)$ and single (59.1\%). The alcohol withdrawal syndrome was the main admission cause $(76.3 \%)$. After leaving the hospital, $70 \%$ of the sample was conducted to aftercare at CAPS$A D$. These results provide preliminary evidence for the need of mental health services emphasizing alcohol dependence problem.

Key words Chemical dependence, Psychiatric admission, General Hospital
Resumo A Unidade de Internação Psiquiátrica do H ospital Geral (UIPHG) éuma proposta articulada ao movimento da reforma psiquiátrica. 0 objetivo deste estudo foi avaliar os determinantes das internações de dependentes químicos na UIPHG Dr. Estevam, em Sobral (CE). Este estudo foi do tipo documental com abordagem quantitativa, envolvendo 203 clientes que foram internados na UIPH G no período de outubro de 2005 a abril de 2006. Os dados foram coletados por meio de um roteiro adaptado a partir do documento usado no momento da internação. O bservou-se predomínio de pacientes do sexo masculino $(95,1 \%)$, com idadeentre 30 e 40 anos $(62,5 \%)$ e solteiros $(59,1 \%)$. Em $76,3 \%$ doscasos, o diagnóstico da internação foi síndrome de abstinência do álcool. A pós alta hospitalar, 70\% desses clientes foram encaminhados ao CAPS-AD. Esses resultados demonstram quão imprescindíveis são os serviços de saúde mental que enfoquem a problemática do alcoolismo.

Palavras-chave Dependência química, Internação psiquiátrica, H ospital Geral 
Introdução

A Unidade de Internação Psiquiátrica em Hospital Geral (UIPHG) representa uma proposta assistencial que se articula ao movimento da reforma psiquiátrica. Este vem propondo alternativas aos hospitais psiquiátricos tradicionais, buscando evitar as internações prolongadas, o hospitalismo, a perda de identidade, de vínculos sociais ede cidadania. A idéia-mestra éoferecer um leque de alternativas que substituam o asilo psiquiátrico, permitindo que as pessoas com transtornos mentais permaneçam o maior tempo possível em suas comunidades de origem ${ }^{1}$.

0 uso indevido de drogas tem sido tratado, na atualidade, como questão de ordem internacional, objeto de mobilização organizada das nações em todo o mundo. Seus efeitos negativos tornam instáveis as estruturas sociais, ameaçam valores políticos, econômicos, humanos e culturais dos estados e sociedades e infligem considerável prejuízo aos países, contribuindo para o crescimento dos gastos com tratamento médico e internação hospitalar, além do aumento dos índices de acidentes de trabalho, de acidentes de trânsito, de violência urbana e de mortes prematuras e, ainda, para a queda de produtividade dos trabalhadores. Afeta homens e mulheres, de todos os grupos raciais e étnicos, pobres e ricos, jovens, adultos e idosos, pessoas com ou sem instrução, profissionais especializados ou sem qualificação. Atinge inclusive, bebês recém-nascidos que herdam doenças e/ou a dependência química de suas mães toxicômanas².

No quesereferea internações, o uso do álcool foi o principal causador deinternações por transtorno mental no país entre 1988 e 1999, respondendo por $90 \%$ dessas, segundo levantamento do Centro Brasileiro de Informações sobre Drogas Psicotrópicas (CEBRID/UNIFESP). A pesquisa também revelou, no mesmo período, um aumento de $4,7 \%$ para $15,5 \%$ na proporção das internações provocadas pela dependência de outras drogas. No período, as internações por uso de cocaína passaram de $0,8 \%$ para $4,6 \%$. Nos dois primeiros anos do trabal ho, 1988 e 1989, a maconha era a droga mais consumida depois do álcool ${ }^{3}$.

No Brasil, o álcool é responsável por $85 \%$ das internações decorrentes do uso de substâncias psicoativas, $20 \%$ das internações em clínica geral e $50 \%$ das internações masculinas psiquiátricas ${ }^{4}$.

É importante ressaltar que, na cidade de Sobral (CE), não há registros oficiais da Secretaria deSaúde dos casos dedependência química, nem de quais os tipos de drogas que estão sendo uti- lizados. 0 que se observa empiricamente é um grande índice de alcoolismo nas comunidades e nas emergências dos hospitais gerais.

N este contexto, ocorreu recentemente em Sobral, município localizado na Região N ortedo Estado do Ceará, uma convergência de esforços governamentais para a implantação de uma rede de atenção à saúde mental que congregasse os diversos equipamentos de saúde existentes no município, em todos os níveis de assistência preconizados 5 .

A RededeAtenção Integral à Saúde M ental de Sobral atualmente é estruturada da seguinte forma: Equipes da Estratégia Saúde da Família (ESF), ambulatório de psiquiatria no Centro de Especialidades M édicas (CEM), Unidade de Internação Psiquiátrica do Hospital Geral (UIPHG), Serviço Residencial Terapêutico (SRT), Centro de Atenção Psicossocial (CAPS) emais recentemente o Centro de Atenção Psicossocial - Álcool e outras Drogas (CAPS-AD).

A pós a implantação dessa nova rede, a atenção à saúde mental em Sobral renasce para promover a saúde e resgatar a cidadania dos portadores de transtornos mentais.

A UIPH G foi criada com o objetivo degarantir uma retaguarda para a urgência psiquiátrica, observação e internação de pessoas com transtorno mental em estado de grave crise psíquica, provenientes do município de Sobral, bem como de todas as cidades da Região Norte do Estado do Ceará aqui referenciadas.

O CAPS-AD foi implantado em setembro de 2002 com o objetivo deatender à demanda específica da dependência química, não só em nível especializado de tratamento, mas também em nível primário de saúde, prevenção, estudo e pesquisa.

Além do ônus social, a dependência química também traz elevados custos financeiros para a sociedade. Segundo o M inistério da Saúde, os gastos com internações psiquiátricas associadas ao abuso de substâncias psicoativas ultrapassaram os valores de $R$ \$ 310 milhões no triênio de 19951996-1997, representando uma das cinco primeiras causas de internação hospitalar no país ${ }^{6}$.

Diante deste quadro em que se encontra o uso e abuso de substâncias psicoativas, com as suas consequências orgânicas, psicológicas e sociais, a UIPH G torna-se um serviço de fundamental importância para o município de Sobral. Atuando não somente no campo do tratamento ereabilitação, mas também em nível primário de saúde, na atenção, preven ção e educação da população acerca da problemática da dependência química, coloca-se em consonância com a Nor- 
matização dos Serviços de Aten ção a Transtorno por U so e Abuso de Substâncias Psicoativas do Ministério da Saúde 6 .

O objeto de estudo mostra-se relevante e original por serem poucas as experiências desta prática de cuidado aos usuários de drogas em UIPHG no Brasil. Sendo assim, pesquisas com esta temática são limitadas.

Com a realização deste estudo, estaremos contribuindo para a construção de uma prática profissional voltada para a realidade identificada, ampliando assim as discussões sobre essa problemática, favorecendo uma prática que valoriza e fortalece a abordagem multidisciplinar e interdisciplinar, consolidando uma rede deserviços de atenção à saúde mental, notadamente aqueles relativos ao uso e/ou abuso de drogas.

0 objetivo deste trabalho foi avaliar os aspectos sociodemográficos e os determinantes clínicos das internações de pessoas com transtornos mentais associados ao uso de substâncias na Unidade de Internação Psiquiátrica do Hospital Geral do Hospital Dr. Estevam no Município de Sobral (CE).

\section{Metodologia}

Este estudo foi do tipo documental com abordagem quantitativa. Os dados foram coletados a partir dos prontuários de 203 clientes acometidos de transtornos mentais associados ao uso de substâncias com registro de internamento na Unidade de Internação Psiquiátrica do Hospital Geral (UIPHG) do Hospital Dr. Estevam no período de outubro de 2005 a abril de 2006. A UIPHG, localizada no Centro da cidade de Sobral (CE), dispõe de dezesseteleitos em enfermaria de psiquiatria e um número variável de leitos na enfermaria de clínica médica - onde, na maioria das vezes, são internados os pacientes com transtornos relacionados ao uso de substâncias psicoativas. Estes leitos são previstos para curta permanência dos usuários, sendo indicados como último recurso para pessoas em crise, cujo atendimento na própria residência ou no serviço ambulatorial não seja possível.

0 instrumento usado para a coleta de dados foi construído a partir do laudo utilizado na rotina hospitalar no momento do pedido de internação. 0 mesmo contemplou as seguintes variáveis: identificação (nome, sexo, data denascimento, estado civil eprocedência) ; dados da internação (data de admissão e de alta, principais sinais esintomas clínicos que motivaram a internação, condições que justificaram a internação, diagnóstico inicial no ato da internação, profissional responsável pelo laudo de internação, se ocorreu reinternação durante o período em estudo e 0 tipo de encaminhamento após a alta hospitalar).

Tivemos várias perdas na coleta de dados dos prontuários, haja vista que havia muitas falhas no preenchimento dos prontuários, como a falta de algumas informações, existência de registros preenchidos de forma ilegível e incoerências nos dados. No total, verificamos 250 prontuários; destes, foram efetivamente contemplados 203 prontuários que continham todas as informações necessárias para realização do estudo.

A pesquisa seguiu os preceitos éticos que regulam a pesquisa com seres humanos (Resolução no 196/96), uma vez que a coleta de dados teve início após pronunciamento favorável da direção da UIPHG Dr. Estevam Ponte, feito via ofício e após o parecer favorável do Comitê de Ética da Universidade Estadual Vale do Acaraú, sendo aprovado com o Certificado de Apresentação para Apreciação Ética - CAAE (0551.0.000.039 - 06). Firmou-se também compromisso de que as informações seriam utilizadas única e exclusivamente para fins acadêmicocientíficos previstos neste estudo.

A organização e o processamento dos dados foram realizados pelo programa Epi-Info (versão 6.04.), seguido de análise quantitativa das questões presentes no instrumento usado para a coleta dos dados.

\section{Análisee discussão dos resultados}

Considerando-se as características sociodemográficas dos clientes, observou-se que $95,1 \%$ deles eram do sexo masculino, na faixa etária entre 30 a 49 anos (62,5\%) e sem vínculos conjugais $(59,1 \%)$. N este contexto, estudos sobre prevalência eincidência do uso dedrogas, embora com divergências nos padrões de consumo entre os países, costumam relatar que o sexo masculino se destaca como o mai or consumidor? ${ }^{7}$.

Com relação à faixa etária, observou-segrandeincidência deinternações nafaseadulta. A idade usual da busca de tratamento para dependência química se dá por volta dos quarenta anos, quando o indivíduo apresenta graves problemas de saúde decorrentes do uso abusivo de álcool ${ }^{8}$.

Resultado semelhante ao desta pesquisa acha-se em Silva et al. ${ }^{9}$, cujos estudos apontam que dois terços dos pacientes internados em hospitais psiquiátricos da cidade do Rio de Janeiro 
eram solteiros e não tinham ou nunca tiveram uma relação conjugal estável.

Com relação à origem dos clientes assistidos na UIPHG Dr. Estevam, 73,4 \% provinham da cidade de Sobral. Os demais $26,6 \%$ vinham de municípios circunvizinhos, sendo que destes $18,5 \%$ eram do município de Forquilha.

Quanto às características específicas da internação, observou-se que o período das internações variou entre quatro equinze dias. Este achado refletea preocupação em não semanter o cliente internado por longos períodos, contrastando com a prática dos antigos hospitais psiquiátricos. Como discutido na III Conferência Nacional de Saúde M ental, deve-se rever o critério de tempo de internação e garantir, por meio de supervisões institucionais e fiscalizações, que ele seja o mais breve possível, considerando a conduta psiquiátrica e avaliação realizada pela equipe multiprofissional que acompanha a pessoa assistida ${ }^{10}$.

Com relação à reinternação, observou-seque apenas 29 clientes $(14,3 \%)$ recorreram a essa prática no período avaliado. Atualmente, acreditase que o ponto-chave para a redução das reinternações seja a disponibilidade de serviços extramurais (ambulatórios, hospitais-dia, oficinas protegidas, etc.) que realizam de forma eficiente a farmacoterapia, a psicoterapia, a orientação, a terapia ocupacional e a reabilitação social ${ }^{1}$.

A Tabela 1 ilustra os principais sinais e sintomas que motivaram a busca por internação na UIPH G Dr. Estevam, sendo observado em 60,6\% dos clientes agitação psicomotora, agressividade, tremores, insônia, alucinações audiovisuais, delírios esudorese.

Os sinais e sintomas de abstinência dependem do tipo de substância usada e aparecem algumas horas ou dias depois quea mesma foi consumida pela primeira vez. No caso dos dependentes deálcool, por exemplo, a abstinência pode ocasionar desde um simples tremor nas mãos a náuseas, vômitos e atéum quadro de abstinência mais grave denominado delirium tremens, caracterizado pela presença de confusão mental, tremores intensos, agitação, hiperatividade autonômica, alterações da cognição e do ciclo sonovigilância, podendo evoluir para o óbito ${ }^{11}$.

A Tabela 2 apresenta a proporção de hipóteses diagnósticas levantadas de acordo com a 10aㅡ Edição da Classificação Internacional de Doenças - CID 10 da OM S. 0 diagnóstico mais frequente foi o de transtorno por uso de substâncias psicoativas, especificamente síndrome de abstinência alcoólica (76,3\%) (CID-10 F- 10.3).

No presente estudo, observou-se que 0 álcool éa droga mais consumida pelos clientes. No Brasil, o alcoolismo constitui-se em um grave problema desaúde pública, uma vez que, deacordo com o Centro Brasileiro de Informação sobre Drogas (CEBRID), essa condição atinge 5 a $10 \%$ da população adulta brasileira ${ }^{12}$.

Com relação às condições que justificaram essas internações, na Tabela 3, observa-se que $55,7 \%$ dos clientes necessitavam de tratamento clínico em virtude de complicações associadas à síndromedeabstinência ao álcool (55,7\%), 12,3\% apresentavam o risco de complicações e $9,4 \%$ necessitavam detratamento psiquiátrico $(9,4 \%)$.

A partir desses resultados, percebe-se que a principal justificativa para a internação foi o surgimento decomplicações clínicas ocasionadas pelo uso abusivo do álcool, sendo a maior parte dessas internações na enfermaria de clínica médica. De acordo com Laranjeira ${ }^{13}$, as complicações clínicas proporcionam um critério da gravidade da dependência e, quando detectadas no início, podem ser tratadas e promover recuperação completa. Além disso, a existência das referidas complicações pode estimular al guns pacientes a buscar a abstinência, aceitando ficar em tratamento.

Tabela 1. Principais sinais e sintomas que determinaram a internação de pessoas com transtornos mentais associados ao uso de substâncias na UIPHG. Sobral (CE).

\begin{tabular}{lrr}
\hline \multicolumn{1}{c}{ Sinais e sintomas } & $\mathrm{N}$ & $\%$ \\
\hline Agitação psicomotora, agressi vidade, tremores, insônia, alucinações audiovisuais, & 123 & 60,6 \\
delírios e sudorese & & \\
Desorientação mental, episódio convulsivo, alucinações audiovisuais e delírios & 42 & 20,7 \\
Tremores, sudorese, vômitos e ansiedade & 31 & 15,3 \\
Outros & 7 & 3,4 \\
Total & 203 & 100,0 \\
\hline
\end{tabular}


Tabela 2. Hipóteses diagnósticas responsáveis pela internação de pessoas com transtornos mentais associados ao uso de substâncias na UIPHG, Sobral (CE), de acordo com 10ā Edição da Classificação Internacional de Doenças - CID 10 da OMS.

\begin{tabular}{lcrr}
\hline \multicolumn{1}{c}{ Diagnóstico } & CID-10 & N & $\%$ \\
\hline Síndrome de abstinência alcoólica & $\mathrm{F} 10.3$ & 155 & 76,3 \\
Dependência de múltiplas drogas & $\mathrm{F} 19.2$ & 14 & 6,9 \\
Dependência de múltiplas drogas + esquizofrenia & $\mathrm{F} 19.2+\mathrm{F} 20.0$ & 5 & 2,4 \\
Intoxicação aguda pelo álcool & $\mathrm{F} 10.0$ & 4 & 2,0 \\
Síndrome de abst. alcoólica com delirium tremens & $\mathrm{F} 10.4$ & 4 & 2,0 \\
Psicose alcoólica & $\mathrm{F} 10.5$ & 4 & 2,0 \\
Síndrome de abst. alcoólica + transtorno bipolar & $\mathrm{F} 10.3+\mathrm{F} 31.2$ & 4 & 2,0 \\
Síndrome de abst. alcoólica + depressão & $\mathrm{F} 10.3+\mathrm{F} 32.3$ & 3 & 1,4 \\
Dependência de Cannabis (maconha) & $\mathrm{F} 12.0$ & 2 & 1,0 \\
Dependência de Cocaína & $\mathrm{F} 14.0$ & 1 & 0,5 \\
Outros & - & 7 & 3,5 \\
Total & - & 203 & 100,0
\end{tabular}

Tabela 3. Distribuição das condições que justificaram a internação de pessoas com transtornos mentais associados ao uso de substâncias na UIPH G. Sobral (CE).

\begin{tabular}{lrr}
\hline \multicolumn{1}{c}{ Condições que justificaram a internação } & $\mathrm{N}$ & $\%$ \\
\hline Necessidade de tratamento clínico & 113 & 55,6 \\
Risco de complicações & 25 & 12,3 \\
Necessidade de tratamento psiquiátrico & 19 & 9,4 \\
Risco para si mesmo & 17 & 8,4 \\
Risco para si eterceiros & 11 & 5,4 \\
Risco devida & 3 & 1,5 \\
Outros & 15 & 7,4 \\
Total & 203 & 100,0 \\
\hline
\end{tabular}

Considerando-se os profissionais médicos responsáveis por essas internações, $75,4 \%$ eram clínicos e 24,6\%, psiquiatras. Como discutido na III Conferência Nacional de Saúde M ental, devese exigir que os municípios implementem o controle de emissão de laudos para internações psiquiátricas por qualquer profissional médico ${ }^{8}$. Vale sal ientar que, duranteo período deinternação, os clientes contam com atendimento clínico sistematizado e diário da psiquiatria, em regime individual e grupal, evolução diária e cuidados gerais da equipe de enfermagem, além do acompanhamento de psicólogos e terapeutas ocupacionais ${ }^{14}$.

Quanto ao tipo de encaminhamento após a alta hospitalar, $70 \%$ dos clientes foram encami- nhados para o CAPS-AD da cidade de Sobral (CE), enquanto que em $14,3 \%$ deles não foi possível localizar qualquer encaminhamento. Embora este fato possa se dever a um cuidado menos sistemático na unidade com os prontuários de pacientes não elegíveis para o tratamento no CAPS-AD, esse achado demanda cuidados.

\section{Conclusões}

O desenvolvimento deste estudo buscou intensificar a discussão em relação aos determinantes das internações de dependentes químicos nas unidades de internação psiquiátrica no Hospital 
Geral. Como as pesquisas que envolvam esses aspectos são mínimas, podemos considerar que foi um desafio o seu desenvolvimento.

Diante dos dados apresentados, iremos expor algumas considerações dentro dos limites que os resultados da amostra permitem concluir, pois ressaltamos que não é a nossa pretensão esgotar toda a discussão que delineia o objeto em pauta.

Podemos perceber que houve um número significativo de internações determinadas pelo 0 uso/abuso do álcool, al go que nos chama a atenção, pois trata-se de uma droga considerada de uso natural, sendo comum o seu consumo no ambiente domiciliar, em festividades ou mesmo em ambientes públicos, sendo a sociedade permissiva ao estimular o consumo da mesma por meio da propaganda, não considerando a de pendência de álcool como uma doença grave, mesmo observando que o seu consumo traz claras consequências.

Diante desse problema, vemos a necessidade de haver uma maior intervenção por parte das políticas públicas na dependência química, não só nas drogas ditas ilícitas, mais principalmente em relação ao álcool, que por ser uma droga lícita, está sendo consumida de forma excessiva ese tornando uma droga perigosa, pois traz consigo várias complicações clínicas, psicológicas, familiares esociais.

Neste estudo, detectamos uma pequena parcela desta problemática. Porém, sabemos que, na realidade, 0 álcool é a substância que mais leva seus usuários à doença e à morte, sendo a que mais causa mortes passíveis de prevenção no mundo. Desse modo, não se trata de absorver, minimizar ou condenar esta ou aquela substância. Todas trazem prejuízos e perigos potenciais que devem ser sempre considerados, independentemente deserem lícitas ou ilícitas.

Podemos afirmar então que os serviços de saúde mental que possuem como características o tratamento e a reabilitação de dependentes químicos são imprescindíveis no nosso meio. N este cenário, devemos destacar a reforma psiquiátrica, movimento quetem orientado as políticas de saúde mental com intuito de qualificar e humanizar os cuidados nesse setor.

0 estudo possibilitou um importante conhecimento do processo deinternação de dependentes químicos na UIPHG, podendo-se com isso apontar estratégias de trabalho mais condizentes com a realidade e políticas mais eficazes na prevenção ao uso abusivo de substâncias psicoativas, bem como na assistência aos dependentes químicos do município.

\section{Colaboradores}

FSP Souza e EN de Oliveira participaram igualmente de todas as etapas da elaboração do artigo. 


\section{Referências}

1. Dalgalarrondo P. Repensando a internação psiquiátrica: A proposta das unidades de internação psiquiátrica de hospital geral [dissertação]. Campinas (SP): Unicamp; 1990.

2. Carlini AE, Galduróz JCF, N oto AR, Nappo SA. I levantamento domiciliar sobre o uso de drogas psicotrópicas no Brasil: estudo envolvendo 107 maiores cidades do pais: 2001. São Paulo: Cebrid/U nifesp; 2002.

3. Noto AR. Álcool provoca $90 \%$ das internações por drogas. [site da Internet]. [acessado em 2002 jun 27]. Disponível em: http://www.unifesp.br/comunicações

4. Galduróz JCF, Noto AR, Fonseca AM, Carlini EA. I Levantamento domiciliar nacional sobre o uso de drogas psicotrópicas - Parte A: Estudo envolvendo as 24 maiores cidades do Estado de São Paulo. São Paulo: Cebrid/Unifesp; 2000.

5. Ferreira-Júnior AR. Por que sou usuário de drogas? Opinião dos adolescentes [monografia]. Sobral (CE): Centro de Ciências da Saúde, Universidade Estadual Vale do Acaraú; 2003.

6. Brasil. Ministério da Saúde. A Política do Ministério da Saúde para Atenção Integral a U suários de Álcool e outras Drogas. 1a ed. Brasília: Ministério da Saúde; 2003.

7. Benigna MJC. Alcoolismo em tratamento. Causas e Consequências. Ciência, Cultura e Saúde 1996; 4:75-83.

8. Schuckit M. Abuso de álcool e drogas. Porto Alegre: Artes M édicas; 1991.

9. Silva JPL, Continho ESF, Amarante PD. Perfil demográfico e socioeconômico da população de internos dos hospitais psiquiátricos da cidade do Rio de Janeiro. Cad Saude Publica [periódico na Internet]. 1999. [acessado 2006 jun 12]; 15(3):[cerca de 7 p.]. Disponível em: www.scielo.br/pdf/csp/v15n3/ 0489.pdf
10. Brasil. Ministério da Saúde. III Conferencia Nacional de Saúde M ental: "Cuidar, sim. Excluir, não. Efetivando a Reforma Psiquiátrica com acesso, qualidade, humanização e controle social. Brasília: Ministério da Saúde, 2002.

11. Secretaria Nacional Antidrogas. Um guia para a família. 4aㅡ ed. Brasília: SENAD; 2003.

12. Laranjeira R, Pinsky I. O alcoolismo. São Paulo: Contexto; 1997.

13. Laranjeira $R$, coordenador. U suários de substâncias psicoativas. Abordagem, diagnóstico e tratamento. $2^{\text {a }}$ ed. São Paulo: Conselho Regional de Medicina do Estado de São Paulo/Associação M édica Brasileira, 2003.

14. Pereira AA, Andrade LOM. Rede integral de atenção à saúde mental de Sobral-CE. In: Lancetti $A$, organizador. Saúde Loucura: saúde mental e saúde da família. 2a ed. São Paulo: Hucitec; 2001.

Artigo apresentado em 05/07/2008

Aprovado em 14/08/2007

Versão final apresentada em 04/12/2007 